\title{
Anti-obesity Effect of Pinellia ternata Extract in Zucker Rats
}

\author{
Yoo-Jeong KIM, ${ }^{a}$ Young-Oh ShIn,,${ }^{b}$ Young-Wan HA, ${ }^{a}$ Sanghyun LeE, ${ }^{c}$ Jae-Keun OH, ${ }^{b}$ and \\ Yeong Shik KIM*,a \\ ${ }^{a}$ Natural Products Research Institute and College of Pharmacy, Seoul National University; Yeongun-dong, Jongno-gu, \\ Seoul 110-460, Korea: ${ }^{b}$ Department of Sports Medicine, Korea National Sport University; Oryun-dong, Songpa-gu, Seoul \\ 138-763, Korea: and ${ }^{c}$ Department of Applied Plant Science, College of Industrial Science, Chung-Ang University; Naw-ri \\ Daeduk-myun, Anseong 456-756, Korea. $\quad$ Received January 10, 2006; accepted February 20, 2006
}

\begin{abstract}
Pinellia ternata is known as the herb effective in removing dampness-phlegm, one of the causes of obesity in traditional Korean medicine. Pinellia ternata water extract (PE) was fed to rats after mixing with diet once a day $\left(400 \mathrm{mg} \cdot \mathrm{kg}^{-1}\right)$ for 6 weeks. We investigated its effect on the thermogenesis and fatty acids oxidation with obese Zucker rats. We also determined the gene expression of uncoupling protein 1 (UCP1), peroxisome proliferatorsactivated receptor $\alpha(\operatorname{PPAR} \alpha)$, and PPAR $\gamma$ coactivator $1 \alpha(\mathrm{PGC} 1 \alpha)$. The PE treatment lowered the levels of triglyceride and free fatty acids $(p<0.05)$ in blood of the obese rats and the body weight was also reduced slightly. It was also observed that PE significantly increased the expression of both UCP1 mRNA in brown adipose tissue (BAT) $(p<0.001)$ and PPAR $\alpha$ and PGC1 $\alpha$ mRNA in white visceral adipose tissue (WAT) $(p<0.05$ and $p<0.001$, respectively), which may cause a reduction of obesity. These results suggested that PE would be able to affect anti-obesity through thermogenesis and fatty acid oxidation.
\end{abstract}

Key words Pinellia ternata; obesity; Zucker rat; peroxisome proliferators-activated receptor $\alpha$; PPAR $\gamma$ coactivator $1 \alpha$; uncoupling protein 1

Metabolic disorders of the spleen, lung and kidney are thought to be the main causes of obesity by practitioners of Oriental medicine. Wind, phlegm, dampness and heat are also possible factors of obesity. ${ }^{1}$ Especially, the unbalanced metabolism in the spleen and stomach caused by heavy drinking, hyperphagia, lycorexia and preference for junk food causes dampness-phlegm. ${ }^{2)}$

Pinellia ternata is one of the herbs effective in removing dampness-phlegm. It invigorates the spleen, resolves phlegm, and removes food stagnation. It is mostly used in the prescription of an herbal formula to treat symptoms due to imbalance of dampness-phlegm. ${ }^{3)}$ Dampness-phlegm is represented by copious amounts of thick sputum or accumulations in the body of thick fluid, which migrates to the flesh, resulting in obesity. ${ }^{3,4)}$

To control obesity, limiting energy intake has made moderate success and energy expenditure must be increased in an obese individual. Energy expenditure is the result of basal metabolism, physical activity, and adoptive thermogenesis. Thermogenesis through the function of UCPs (uncoupling proteins) is very important in obesity. ${ }^{5)}$ As its name suggests, it uncouples respiration from ATP synthesis by dissipating the transmembrane proton gradient as heat. ${ }^{6)}$ UCP is therefore, commonly recognized as a key molecule in metabolic thermogenesis and its dysfunction contributes to the development of obesity. ${ }^{7)}$ So far three main isoforms of UCP have been reported. UCP1 is expressed exclusively in brown adipose tissue (BAT) and UCP2 is present in various organs, while UCP3 is expressed in skeletal muscle. ${ }^{8)}$ The best-characterized member of the mitochondrial transporter superfamily involved in energy expenditure and brown fat thermogenesis is thermogenin or UCP1.9) UCP gene expression is upregulated by ligands for nuclear receptors such as thyroid hormone receptor, peroxisome proliferator-activated receptors (PPARs) and retinoid-X receptor. ${ }^{10)}$

PPARs are members of the superfamily of nuclear transcription factors regulating lipid metabolism, also referred as fatty acid activated transcription factors. ${ }^{11)}$ There are three members: $\operatorname{PPAR} \alpha, \operatorname{PPAR} \gamma$, and $\operatorname{PPAR} \beta / \delta$, with different tissue distribution. PPAR $\alpha$ plays an essential role in maintaining lipid homeostasis in the liver and the heart by modulating both constitutive and inducible expression of genes that regulate fatty acid catabolism. ${ }^{12,13)}$ It is also expressed in tissues that are characterized by high rates of fatty acid oxidation. $^{13)}$

PPAR coactivator $1 \alpha(\mathrm{PGC} 1 \alpha)$ participates in the transcription of important genes for energy metabolism. The basic role of $\mathrm{PGC} 1 \alpha$ is the transcriptional regulation of both coupled and uncoupled metabolism. ${ }^{14)} \mathrm{PGC} 1 \alpha$ stimulates mitochondrial biogenesis and respiration in muscle cells through an induction of UCP and regulation of the nuclear respiratory factors. ${ }^{15,16)}$ Therefore, PPAR $\alpha$ and PGC $1 \alpha$ are known to be important transcription factors in fatty acid oxidation and mitochondrial biogenesis. ${ }^{17)}$

In our study, we examined the anti-obesity effect of supplementation of $P$. ternata extract (PE) on gene expression of UCP1, PPAR $\alpha$, and PGC1 $\alpha$ in adipose tissues (brown and visceral) of obese Zucker rats and determined the biochemical parameters including body weight, the levels of total cholesterol, triglyceride, and free fatty acid in the blood.

\section{MATERIALS AND METHODS}

Preparation of PE Crude drugs (Pinellia ternata TENORE et BREIT.) originated in North Korea were identified by Prof. Ho-Chul Kim (Department of General Herbal Pharmacology, College of Oriental Medicine, Kyung Hee University). P. ternata $(157 \mathrm{~g})$ was decocted in a round flask with distilled water $(3000 \mathrm{ml})$ at $100{ }^{\circ} \mathrm{C}$ for $2 \mathrm{~h}$ and filtered. Distilled water $(1000 \mathrm{ml})$ was then added and decocted at $100^{\circ} \mathrm{C}$ for $1 \mathrm{~h}$ and filtered again. The filtrate was reduced and freezedried as a powder with $24.2 \%$ yield ( $38 \mathrm{~g}$ ).

Animals Male obese (HsdHlr:Zucker-fa, $n=12$ ) and lean Zucker (HsdHlr:Zucker-lean, $n=6$ ) rats were purchased 
(6 weeks of age) from Harlan (Indianapolis, U.S.A.). Animals were housed alone under controlled light (12-h light and dark cycle) and temperature $\left(22 \pm 2{ }^{\circ} \mathrm{C}\right)$ conditions. Animals had access to food and water ad libitum. After an adaptation period of 2 weeks, the animals were divided into lean, obese control, and obese treatment groups. Each group consisted of 6 rats and all animals were fed a normal diet. The obese treatment group was administered PE at a daily-dose of $400 \mathrm{mg} \cdot \mathrm{kg}^{-1}$ for 6 weeks. The control group was administered distilled water daily. After the experiment period, animals were anesthetized and the interscapular brown adipose tissue (BAT) and white visceral adipose tissue (WAT) were removed.

Plasma Biochemical Analysis Blood was withdrawn from the femoral artery, centrifuged at $900 \boldsymbol{g}$ for $15 \mathrm{~min}$ and the plasma was obtained. Alanine aminotransferase (ALT), aspartate aminotransferase (AST), total cholesterol (TC) and triglyceride (TG) were measured using Roche Diagnostic reagents on Cobas Mira analyzer (Roche Diagnostic Pty Ltd.). Free fatty acids (FFA) were measured using an enzymatic assay kit (NEFA C kit; Wako Chem., Japan).

Real Time PCR Total RNA was isolated using RNAzol Bee (Tel-Test, U.S.A.). First-strand cDNA was generated from $2 \mu \mathrm{g}$ of total RNA using a Ready-To-Go T-Primed FirstStrand kit (Amersham Biosciences). Primer and probes were designed using Primer Express software package version 2.0 (Applied Biosystems, Foster City, CA, U.S.A.) from gene sequences obtained from GenBank (UCP1 NM_012682; PGC$1 \alpha$, NM_031347; PPAR $\alpha$, NM_013196).

PCR was performed using the ABI PRISM 7900HT sequence detection system (Applied Biosystems). To compensate for variations in input RNA amounts and efficiency of reverse transcription, GAPDH (NM_017008) mRNA was quantified, and results were normalized to these values. The relative expression of the gene of interest was calculated and reported as arbitrary units.

Quantification of Ephedrine from PE Approximately $1.0 \mathrm{~g}$ of the water extract was added to a round-bottomed flask containing $20 \mathrm{ml}$ of distilled water and the mixture was shaken at room temperature for $6 \mathrm{~h}$. After centrifugation for $30 \mathrm{~min}$ at $1000 \boldsymbol{g}$, the supernatant was freeze-dried and the dried material was dissolved in $2 \mathrm{ml}$ of water. Twenty microliters of the sample was loaded onto a Hitach L-6000 instrument equipped with a UV detector (Spectra-Physics Spectra 100) and an Agilent Zorbax SB-Aq $\mathrm{C}_{18}$ column (4.6 mm I.D. $\times 150 \mathrm{~mm}$, particle size $5 \mu \mathrm{m})$. HPLC conditions were as follows: eluent A, $10 \%$ acetonitrile in water containing $0.4 \%$ SDS; eluent $\mathrm{B}, 35 \%$ acetonitrile in water containing $0.4 \% \mathrm{SDS}$; gradient, $0-20 \mathrm{~min}(0-100 \% \mathrm{~B}), 20-40 \mathrm{~min}$ $(100 \% \mathrm{~B})$, and then equilibrated with $0 \% \mathrm{~B}$ for $10 \mathrm{~min}$. The flow rate was $1 \mathrm{ml} / \mathrm{min}$ and the effluent was monitored at $210 \mathrm{~nm}$.

Statistical Analysis All data were presented as the mean \pm S.E. Statistical comparisons were made using analysis of variance (ANOVA) by Bonferroni's post hoc comparison. A value of $p<0.05$ was considered statistically significant.

\section{RESULTS AND DISCUSSION}

The beneficial effect of PE is clearly visible from the blood biochemical profiles. The data in Table 1 show that PE
Table 1. Determination of Biochemical Parameters after 6 Weeks in Blood

\begin{tabular}{lccc}
\hline \hline \multicolumn{1}{c}{ Parameter } & Lean $(n=6)$ & Obese $(n=6)$ & PE $(n=6)$ \\
\hline Body weight $(\mathrm{g})$ & $361.67 \pm 18.40$ & $503.00 \pm 9.13$ & $485.00 \pm 13.05$ \\
ALT $\left(\mathrm{U} \cdot 1^{-1}\right)$ & $89.00 \pm 4.15$ & $100.40 \pm 11.74$ & $102.80 \pm 9.64$ \\
AST $\left(\mathrm{U} \cdot 1^{-1}\right)$ & $64.50 \pm 2.54$ & $96.40 \pm 12.09$ & $96.40 \pm 4.18$ \\
TC $\left(\mathrm{mg} \cdot \mathrm{dl}^{-1}\right)$ & $90.00 \pm 3.63$ & $146.00 \pm 3.92$ & $146.80 \pm 2.24$ \\
TG $\left(\mathrm{mg} \cdot \mathrm{dl}^{-1}\right)$ & $137.50 \pm 5.10$ & $583.60 \pm 28.31$ & $440.20 \pm 24.78^{* *}$ \\
FFA $\left(\mathrm{mmol} \cdot 1^{-1}\right)$ & $0.234 \pm 0.010$ & $0.542 \pm 0.051$ & $0.421 \pm 0.056^{*}$ \\
\hline
\end{tabular}

Lean: lean control rats, Obese: obese control rats, PE: P. ternate extract treatment obese rats. Data are the mean \pm S.E. Significantly different from obese groups $(* p<0.05, * * p<0.01)$. ALT: alanine aminotransferase, AST: aspartate aminotransferase, TC: total cholesterol, TG: triglyceride, FFA: free fatty acids.

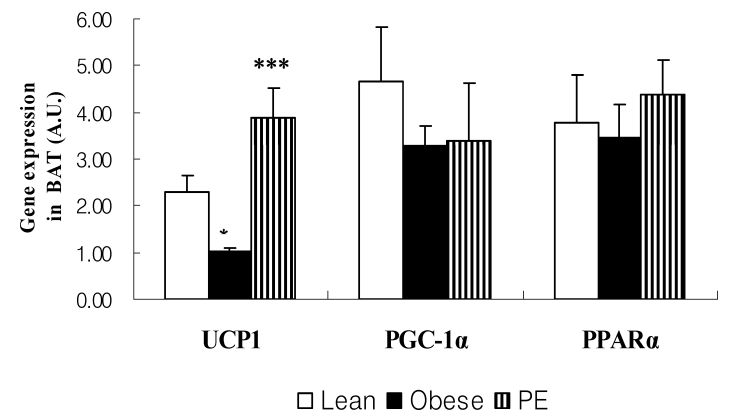

Fig. 1. UCP1, PGC1 $\alpha$ and PPAR $\alpha$ mRNA Expression Was Measured in BAT of Zucker Rats ( $n=6 /$ Group)

Significantly different from the lean groups $(* p<0.05)$. Significantly different from the obese groups $(* * * p<0.001)$. A.U.: arbitrary unit. PE: P. ternate extract.

significantly improves plasma TG level $(p<0.01)$ and FFA level $(p<0.05)$. There was no significant difference in body weight between the obese control group and the PE treated group. After the 6 week experiment, the effect of genotype (lean vs. obese) on daily food intake was as follows: $25.12 \pm$ $0.70 \mathrm{~g} / \mathrm{d}$ in lean rats $v s .38 .80 \pm 1.02 \mathrm{~g} / \mathrm{d}$ in obese rats $(p<$ $0.001)$, vs. $37.0 \pm 1.05 \mathrm{~g} / \mathrm{d}$ in PE-treated rats $(p<0.001)$. There was no significant difference in daily food intake between obese and PE-treated rats, even though the intake showed a 4.64\% $(p=0.599)$ greater downward trend in PEtreated groups than in obese control; this was similar to body weight changes.

The thermogenic effect of PE has gained strong interest because of expectations of its possible contribution to antiobesity. The PE treatment induced the expression of UCP1 mRNA in BAT to increase by 3.78-fold, but no differences were noted in the expression of mRNA for PPAR $\alpha$ and PGC1 $\alpha$ (Fig. 1); in WAT the PE treatment group showed a drastic increase in mRNA expression of PPAR $\alpha$ (9.42-fold) and PGC1 $\alpha$ (6.57-fold) (Fig. 2).

UCP1 expression is an important factor in regulating energy balance and it is modulated by diet and metabolic hormones such as leptin and glucocorticoids. UCP1 is found exclusively in brown adipose tissue, composed of multilocular lipid storage cells that play a crucial role in non-shivering thermogenesis. ${ }^{6,18)}$ Koza et al. investigated the relationship of UCP-1 in adipose tissue with heat production by thermal signature analysis. ${ }^{17)}$ Brown adipose tissues comprise a very large number of mitochondria packed with cristae, densely innervated by the sympathetic nervous system (SNS). Noradrenaline (NA) from sympathetic nerve endings is released in the proximity of brown fat cells. ${ }^{19)}$ NA binds $\beta 3$-adrenergic receptors on the fat cell surface, which initiates a cascade 


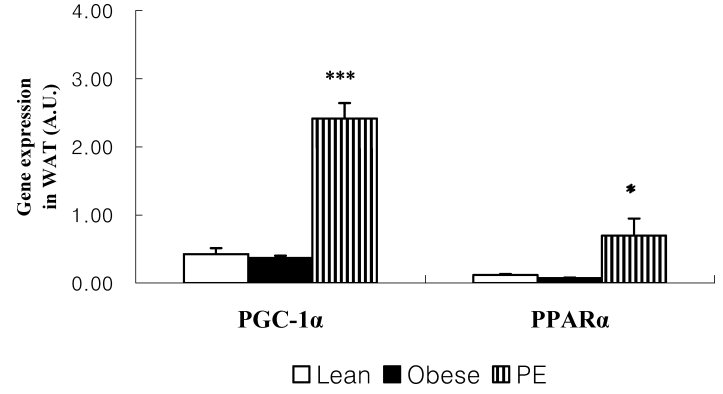

Fig. 2. PGC1 $\alpha$ and PPAR $\alpha$ mRNA Expression Was Measured in WAT of Zucker Rats ( $n=6 /$ Group)

Significantly different from the obese groups $(* p<0.05$, $* * * p<0.001)$. A.U.: arbitrary unit. PE: P. ternate extract.

of metabolic events including the activation of UCP1. ${ }^{20)}$ The agonist of $\beta 3$-adrenergic receptors prevents or reverses obesity. ${ }^{6)}$ In our results, the PE treatment induced a significant 3.78-fold increase in the expression of UCP1 mRNA in BAT, but the level of UCP1 mRNA in WAT was negligible due to its low sensitivity. These results were confirmed in the expression of protein level through Western blot analysis. The protein expression was very small and not different from the control (data not shown). This result is consistent with the previous report that the UCP protein is not present in WAT. ${ }^{20}$ )

Our observations suggest that supplementation of PE induced an increase thermogenesis in BAT and fatty acid oxidation in WAT. This result would create a negative energy balance and subsequent loss of fat content within these tissues.

$\operatorname{PPAR} \alpha$ and $\operatorname{PGC} 1 \alpha$, transcription factors that regulate genes involved in fatty acid oxidation and mitochondrial biogenesis were observed in BAT and WAT. The increased expression of PPAR $\alpha$ and PGC1 $\alpha$ genes in WAT was observed, while no difference was noticed in the expression of mRNA for PPAR $\alpha$ and PGC $1 \alpha$ in BAT (Fig. 1). Within WAT the PE treatment group showed a significant increase in mRNA expression of PPAR $\alpha(0.70 \pm 0.25$ vs. $0.07 \pm 0.01$ : 9.49-fold, $p<0.05), \quad \mathrm{PGC} 1 \alpha(2.42 \pm 0.23$ vs. $0.37 \pm 0.03$ : 6.57-fold, $p<0.001$ ) compared with control (Fig. 2). The PGC1 $\alpha$ highly expressed in WAT was capable of preventing fat from storing and it affected the UCP1 expression in BAT. As a result, $\operatorname{PPAR} \alpha$ and $\operatorname{PGC} 1 \alpha$ were down-regulated in the BAT of obese rats. Following our experiment, we recognized that the alteration of energy balance through an increased utilization of WAT may be conceivable for the treatment of obesity.

Koza et al. reported that PPAR $\alpha$ and $\mathrm{PGC} 1 \alpha$ were more highly expressed in WAT $\beta 3$-adrenoceptor agonist CL-fed NZO mice than in NZO control animals. ${ }^{17)}$ Their observations suggest that inguinal and retroperitoneal fat depots have at least converted into thermogenically active BAT, thus being capable of utilizing fatty acids production via uncoupling of oxidation phosphorylation. These changes would create a negative energy balance and subsequent loss of fat content within these tissues.

Pinellia sp. is one of the plants used as a source containing ephedrine, ${ }^{21,22)}$ including $\beta$-sitosterol, and choline. ${ }^{22)}$ It has been suggested that ephedrine may have a sedative effect rather than stimulant property, which might be quite opposite to expectation on ephedrine, ${ }^{21,23)}$ however the content of this component is very small $(0.002 \%){ }^{21)}$ Ephedrine is used for obesity treatment, which is known to have a thermogenic effect via stimulation of $\beta$-adrenaline receptors and release of noradrenaline from sympathetic nerve endings. ${ }^{24)}$ We treated $1.2 \mu \mathrm{g}$ ephedrine in $1 \mathrm{mg} \mathrm{PE}(0.012 \%)$, and which is equvalent to the amount of $0.48 \mu \mathrm{g}$ ephedrine fed to the obese Zucker rats $(\mathrm{kg})$. There is no effect of PE on animal behavior during experimental period.

To ascertain whether PE induced hepatic damage, serum catalytic concentrations of ALT and AST levels were also measured. A significant $(p<0.05)$ increase in AST levels was observed in plasma from the obese rats (obese, PE) compared with lean control rats. The levels of ALT and AST did not change with PE treatment. This data suggests that PE does not exert any appreciable hepatic toxicity. As previously reported, the repeated-dose toxicity of PE in male and female Sprague-Dawley rats also did not exert considerable toxic effects. $^{25)}$

In conclusion, PE leads the white fat depots to convert into thermogenically active BAT, at least partially, which is capable of utilizing fatty acids for production of heat via uncoupling of oxidative phosphorylation. These changes would create a negative energy balance and subsequent loss of fat content within these tissues. It suggests that PE also protect, against fat storage and may have an anti-obesity effect.

Acknowledgments We thank Kwangdong Pharmaceutical Co. for supplying $l$-ephedrine hydrochloride and Dr. Hai Lin Zhao for helpful discussion.

\section{REFERENCES}

1) Kang Y. Y., J. Beijing Chin. Medical College, 8, 26 (1995).

2) Jin K.Y., Beijing Chinese Med. Pub., 1991, 682-686 (1991).

3) Lee S. J., “Ben Cao Gang Mu,” Ilzungsa, Seoul, 1991, pp. 1192 1201.

4) Chen S. D., "Dan-Shi-Bi-Lu,” Haenglimseowon, Seoul, 1982, p. 76.

5) Bray G. A., Tartaglia L. A., Nature (London), 404, 672-677 (2000).

6) Lowell B. B., Spiegelman B. M., Nature (London), 404, 652-660 (2000).

7) Klingenberg M., Huang S., Biochim. Biophys. Acta, 1415, 271-294 (1999).

8) Cortright R. N., Zheng D., Jones J. P., Fluchey J. D., Dicarlo S. E., Grujic D., Lowell B. B., Dohm G. L., Am. J. Physiol., 276, 217-221 (1999).

9) Himms-Hagen J., J. Nutr. Rev., 41, 261-267 (1983).

10) Saito M., Ohashi A., Nippon Yakurigaku Zasshi, 118, 327-333 (2001).

11) Willson T. M., Brown P. J., Sternbach D. D., Henke B. R., J. Med Chem., 43, 527-550 (2000).

12) Gilde A. J., Bilsen M. V., Acta Physiol. Scand., 178, 425-434 (2003).

13) Braissant O., Foufelle F., Scotto C., Dauca M., Wahli W., Endocrinol., 137, 354-366 (1996).

14) Hammarstedt A., Jansson P. A., Wesslau C., Yang X., Smith U., Biochem. Biophy. Res. Commun., 301, 578-582 (2003).

15) Puigserver P., Spiegelman B. M., Endocrinol. Rev., 24, 78 -90 (2003).

16) Wu Z., Puigserver P., Andersson U., Zhang C., Adelmant G., Mootha V., Troy A., Cinti S., Lowell B., Scarpulla R. C., Spiegelman B. M., Cell, 98, 115-124 (1999).

17) Koza R. A., Flurkey K., Dawn M., Graunke D. M., Braun C., Pan H. J., Reifsyder P. C., Kozac L. P., Leiter E. H., Metabolism, 53, 799-808 (2004).

18) Himms-Hagen J., FASEB J., 4, 2890-2898 (1990)

19) Clapham J. C., Arch J. R. S., Tadayyon M., Pharmacol. Ther., 89, 81121 (2001).

20) Kim H. K., J. Kor. Soc. Study Obes., 2, 14-22 (1993). 
21) Oshio H., Tsukui M., Mstsuoka T., Chem. Pharm. Bull., 26, 2096 2097 (1978)

22) Kim H. C., “ Herbal Pharmacology,” Jipmoondang, Korea, 2001, pp. $346-347$.

23) Mao S. J., Wu L. Y., Cheng L. O., J. Chin. Herbal Drugs, 19, 218
220, 256 (1994).

24) Liu Y. L., Toubro S., Astrup A., Stock M. J., Int. J. Obes., 19, 678685 (1995).

25) Lee J. E., Kim H. J., Choi E. K., Chai H. Y., Yun Y. W., Kim D. J., Kor J. Lab. Anim. Sci., 19, 127-141 (2003). 